\title{
Recovering or Resolving
}

National Cancer Institute

\section{Source}

National Cancer Institute. Recovering or Resolving. NCI Thesaurus. Code C49496.

One of the possible results of an adverse event outcome that indicates that the event is improving. 